\title{
MICROWAVE CHARACTERIZATION OF THE WAVEGUIDE BPM
}

\author{
$\underline{\text { T. Kamps }}^{*}$, R. Lorenz, DESY Zeuthen, Zeuthen, Germany \\ S. deSantis ${ }^{\dagger}$, LBNL, Berkeley, USA
}

\section{Abstract}

For electron beam orbit observation at the TESLA Test Facility FEL one undulator module will be equipped with ten waveguide-type beam position monitors having a design frequency of $12 \mathrm{GHz}$. Since the operation of this waveguide BPM is related to microwave concepts, a precise characterization of its microwave behaviour is necessary to describe and optimize the system performance. The purpose of this paper is to summarize the theory, different simulations and measurements of the device. Calculations and MAFIA simulations are presented for the coupling into the ridged waveguide as well as for the design of the waveguide-to-coax transition. Beside that the resonant frequencies of trapped modes inside a waveguide are estimated. Finally, back reactions on the electron beam in terms of wakefields are estimated by numerical simulations and by a simple analytical model.

\section{MOTIVATION}

The Free Electron Laser (FEL) at the TESLA Test Facility (TTF) [1] is designed for a radiation in the spectral band from VUV to soft X-rays. To ensure the overlap between photon and electron beam, the undulator modules will be equipped with beam position monitors (BPM) and correctors. For the BPMs, the realization of two different system concepts with small vertical dimension is under way [2]. Beside that, diagnostic ports between two adjacent modules are foreseen. A BPM system consists of a transducer close

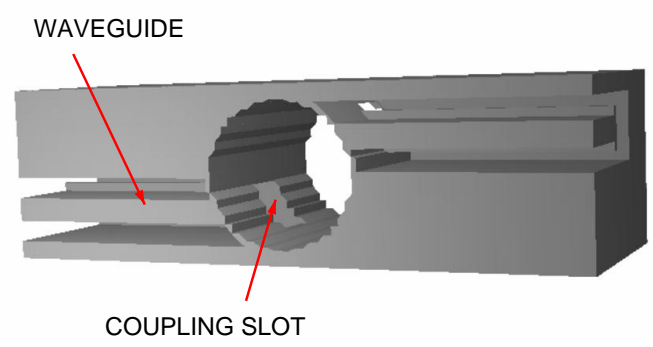

Figure 1: One BPM plane with two waveguides.

to the beam, transmission lines, signal processing electronics, and control software. This paper concentrates on the

\footnotetext{
*Email: tkamps@ifh.de

† Supported in part by Department of Energy contract DE-AC0376SF00098
}

microwave components close to the beam realizing the coupling to the magnetic field, and the transmission to the $50 \Omega$ system outside the vacuum chamber. Fig.1 shows the first part of the transmission line for two waveguide channels.

\section{GENERAL BPM CONCEPT}

Because of limited space in vertical direction and due to short-bunch operation, a microwave concept was considered for a BPM system. With four slots and waveguides a position sensitive signal from the electro-magnetic field of the electron beam will be detected. Because of space limits one BPM consists of two waveguide pairs grouped in planes separated in beam direction. One BPM channel with the objects discussed in this paper is sketched in Fig.2. The

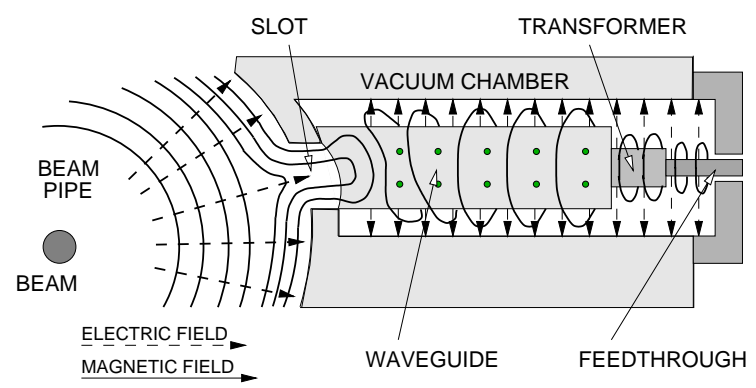

Figure 2: Zoom into vacuum chamber with one BPM channel and electro-magnetic field distribution.

magnetic field penetrates through a slot into the waveguide. The amplitude of the excited fundamental mode is directly proportional to beam offset. To enable a good coupling to the vacuum feedthrough a short rectangular coaxial line follows the waveguide, forming an impedance transformer.

\section{MICROWAVE DESIGN ASPECTS}

For the design, the whole structure was divided into two parts: The one which determines the coupling to the beam - and, hence, the induced signal strength - and the adapter to the $50 \Omega$ coaxial cable system. Technological aspects were taken into account in all design steps.

First it is important to estimate the signal, which will be down-converted and detected in a heterodyne receiver.

\subsection{Slot coupling}

Intuitively the slot coupling mechanism can be described in terms of pertubated wall currents. As the slot cuts the way of the wall currents, energy by means of magnetic field can penetrate into the region behind the slot. For good BPM 
performance, a coupling of $1 \%$ was sighted to obtain a reasonable compromise between signal to noise ratio and interference with the beam. Driving parameters for the coupling are geometry as well as slot size and location in the waveguide profile. Transmission from beam pipe to one waveguide port was simulated using the MAFIA package [3], and the model is shown in Fig.1.

As computer simulations are time consuming an attempt was made to describe analytically the slot coupling by using Bethe's theory [4]. To evaluate the coupling impedance it is necessary to calculate the fields induced in a vacuum chamber for a given current distribution. First, one finds the fields produced by the current distribution without the slot. Then, these fields are considered as an electromagnetic wave on the slot. In the next step it is obvious to find the fields diffracted by the slot into the waveguide. These diffracted fields bear a resemblance with fields radiated by equivalent electric or magnetic dipoles, whose moments are proportional to the normal electric field and the tangential magnetic field of the incident wave. After comparing these fields one obtains the coupling impedance. As a first step a simple structure, where a rectangular waveguide is positioned with its longitudinal axis normal to a cylindrical beampipe surface (see Fig.3), has been investigated using a modified Bethe theory [5]. According to this the ratio of the magnetic fields of the fundamental mode $H_{x}$ and of the beam $H_{\psi}$ is

$$
\left|\frac{H_{x}}{H_{\psi}}\right|=\frac{2 \tilde{\alpha}_{m} \frac{k_{z}}{a b}}{\sqrt{1+\left(2 \tilde{\alpha}_{m} \frac{k_{z}}{a b}\right)^{2}}}
$$

where $a$ and $b$ are waveguide dimensions and $k_{z}$ wave vector pointing downwards the waveguide. To include the effect of the coupling aperture thickness $T$, the thick wall polarizibility $\tilde{\alpha}_{m}$ is needed, given by

$$
\tilde{\alpha}_{m}=0.67 \alpha_{m} \exp \left(-\frac{\pi}{w} T\right) \quad \text { with } \quad \alpha_{m}=\frac{\pi}{16} l w^{2}
$$

for the magnetic polarization of a rectangular slot with edge lengths $l$ and $w$. A comparison between MAFIA simulations for $S_{21}$ and field ratio calculations according to Eqs.1 and 2 is shown in Fig.3, for a standard X-band waveguide coupling via a $4 \mathrm{~mm} \times 4 \mathrm{~mm}$ slot to a beam pipe. For frequencies above the waveguide's cut-off the deviation between MAFIA and theory is less than 5\%. Further studies will investigate the BPM case with its ridged waveguide.

\subsection{Ridged waveguide}

Transverse space limitations and the vacuum constraints of no extra material inside the vacuum chamber required special interest for the waveguide design.

The waveguide has been made ridged to reduce its size. In addition, the hammer-like shape concentrates the magnetic field lines at the location of the coupling slot, so that they are parallel to the magnetic field of the beam over the entire slot.

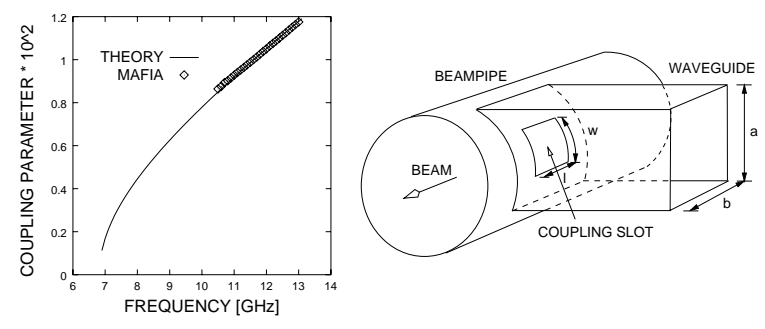

Figure 3: Left: Comparision chart for MAFIA $S_{21}$ simulations against analytical calculations. Right: Geometry used for calculations.

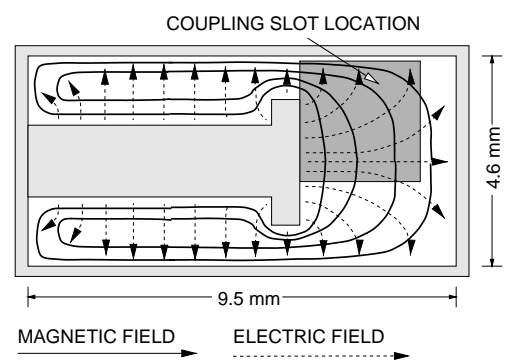

Figure 4: Profile cut of the waveguide with electromagnetic field distribution of the fundamental mode.

The working frequency was limited because of the $\mathrm{H}_{10}$ cut-off frequency treating the beam pipe as a circular waveguide. Keeping this in mind, the working frequency and thus the frequency for the first stage of the signal processing electronics was set to $\nu_{w}=12 \mathrm{GHz}$. Commercially available electronics components from satellite technology in the X-band can be used for signal processing. Size and shape of the waveguide (see Fig.4) have been optimized for a coupling of about $1 \%$. With the present design it is possible to extrude the waveguide structure in one step by electro-discharge machining (EDM).

\subsection{Waveguide-to-coax transition}

The signal excited in the waveguide has to be coupled into a $50 \Omega$ coaxial cable to be detected. An ultra-high vacuum microwave feedthrough by KAMAN Corp. (SMA connector, part \#853872) was foreseen for this purpose. In a first design an electrical coupling was chosen. The resulting adapter was very narrowband, difficult to be tuned to $12 \mathrm{GHz}$, and the welding into special flanges caused problems. In a new design a smooth transition from the waveguide into the feedthrough has been realized by a $\lambda / 4$ transformer, formed by a rectangular coaxial line. This new geometry shown in Fig. 5 for prototype II results in a closer spacing of both planes in beam direction, has larger bandwidth (no tuning problems), and is better for fabrication and installation. The design realized in the vacuum chamber follows the idea sketched for prototype II. 

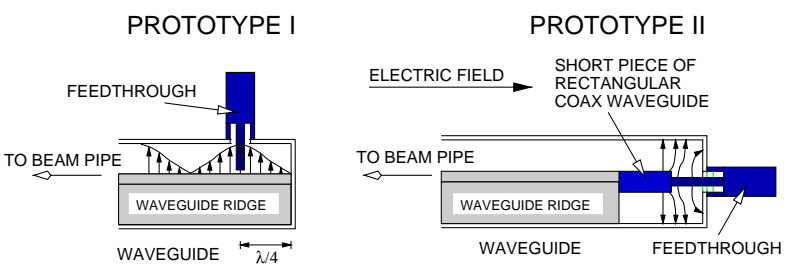

Figure 5: Waveguide-to-coax transitions, first and improved design.

\section{INTERFERENCES}

\subsection{Resonances inside the Detector}

Resonances of trapped modes inside the waveguide close to the working frequency enhance the signal amplitude, but due to fabricational problems it would be difficult to tune and stabilize all waveguides to the same resonance frequency.

Waves beyond $\nu_{c}=8.99 \mathrm{GHz}$ can propagate into the waveguide and excite resonant modes there. Every waveguide of length $l$, which is not perfectly matched at the both ends, has $q$ longitudinal resonances according to

$$
\nu_{r}^{2}=\nu_{c}^{2}+\frac{c_{o}^{2}}{4}\left(\frac{q}{l}\right)^{2}
$$

Two different prototypes and one undulator vacuum chamber with ten BPMs were built.

Tab. 1 compares calculated and measured modes for the second prototype and for the undulator vacuum chamber. The length of both structures estimated from the design are $44.9 \mathrm{~mm}$ and $20.6 \mathrm{~mm}$, respectively. Since it is difficult to include the wall curvature and the electrical length of the adapter into the resonance condition, measured values deviate sligthly from calculated ones using Eq.3.

\begin{tabular}{|c|c|c|c|c|}
\hline \multirow{3}{*}{ Mode \# } & \multicolumn{4}{|c|}{ Resonance [GHz] } \\
\hline & \multicolumn{2}{|c|}{ Prototype II } & \multicolumn{2}{|c|}{ Vacuum chamber } \\
\hline & Estimate & Measure & Estimate & Measure \\
\hline $1 / 2$ & 11.23 & 11.32 & 17.17 & 17.72 \\
\hline $1 / 3$ & 47 & 13.30 & \multicolumn{2}{|c|}{ above cut-off } \\
\hline $2 / 1$ & 13.67 & 13.60 & 15.14 & 15.32 \\
\hline $2 / 2$ & 14.84 & 14.65 & \multicolumn{2}{|c|}{ above cut-off } \\
\hline
\end{tabular}

Table 1: Estimated and measured resonances. Mode \# designates the cut-off number and the parameter $q$; values for the vacuum chamber are averaged over all BPMs.

\subsection{Wakefields}

The coupling slot act as a discontinuity in the beam pipe and will therefore excite transversal and longitudinal wakefields. Although energy is radiating into the slot, the diffraction model [6] can be used to estimate the longitudinal peak wake potential. Fig.6 shows the longitudinal wake potential for a bunch length of $\sigma=250 \mu \mathrm{m}$, and values for the peak wake potential for different bunch lengths. Both were simulated using MAFIA.
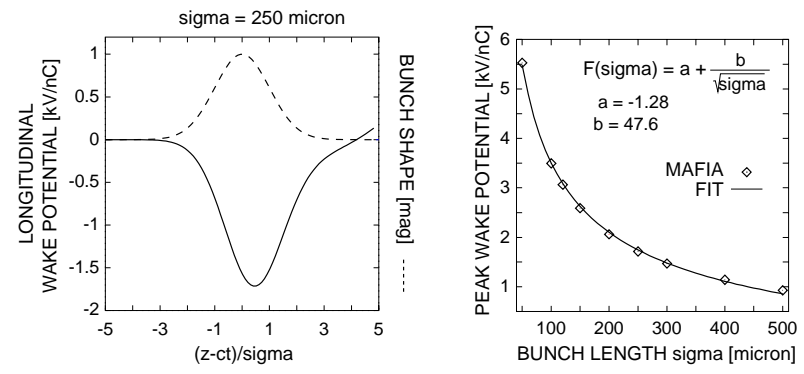

Figure 6: Left: Wake Potential simulated using MAFIA. Right: Diffraction model fitted to simulations for different bunch lengths.

\section{CONCLUSION}

Several attempts have been followed to describe the microwave properties of the waveguide BPM. Simulations using MAFIA in comparison with theoretical models have been proven useful to qualify and optimize the system performance.

\section{ACKNOWLEDGEMENTS}

The authors would like to thank U.Hahn, G.Schmidt and the TESLA group at Zeuthen for many fruitful discussions.

\section{REFERENCES}

[1] A VUV Free Electron Laser at the TESLA Test Facility Linac - Conceptual Design Report, DESY Hamburg, TESLA-FEL 95-03, 1995

[2] R. Lorenz, T. Kamps and M. Wendt, Beam Position Measurement Inside the FEL-Undulator at the TESLA Test Facility Linac, presented at the DIPAC97, Frascati, 1997

[3] R. Klatt et al., MAFIA - A Three-Dimensional Electromagnetic CAD System for Magnets, RF Structures and Transient Wake-Field Calculations, IEEE Proceedings of the LINAC 86, p. 276

[4] H.A. Bethe, Theory of diffraction by small holes, Phys. Rev., Vol.66, No.7 and 8, 1944,pp.163-182

[5] S. deSantis, Radiation effects of slots and coupling holes in the vacuum chamber of particle accelerators, $\mathrm{PhD}$ thesis, University of Rome 'La Sapienza', 1998

[6] K.L.F. Bayne, M. Sands, Wakefields of Very Short Bunches in an Accelerating Cavity, SLAC-PUB-4441, 1987 